\title{
Repräsentationen des Globalen Südens in der religiösen Bildung am Beispiel des Schulbuchs „Kursbuch Religion“
}

\begin{abstract}
Zusammenfassung
Religiöse Bildung in einer globalisierten Welt findet unter anderem mithilfe von Repräsentationen statt. Die Religionsbuchreihe „Kursbuch Religion“ als Mikrokosmos wird auf ihre Repräsentationen des Globalen Südens unter Bezug auf postkoloniale Theorien in diesem Beitrag näher in den Blick genommen. Zum einen werden diese Ergebnisse diskutiert, zum anderen wird ihr Anregungspotenzial für postkoloniale Repräsentationen in der religiösen Bildung sowie für Repräsentationen des Makrokosmos Religion im Kontext Globalen Lernens nachgezeichnet.
\end{abstract}

Schlüsselworte: Repräsentation, Globaler Süden, Postkolonialismus, Schulbuchforschung

\begin{abstract}
Religious Education in a globalised world takes place - among others - with the help of representations. In relation to postcolonial theories a series of the Religious Education textbook "Kursbuch Religion" as a microcosm is analysed in its representations of the Global South. On the one hand, results are discussed; on the other hand, its motivating potential for postcolonial representations in Religious Education as well as representations of the macrocosm religion in the context of global learning are portrayed.
\end{abstract}

Keywords: Representation, Global South, Postcolonialism, Textbook Research

\section{Überblick}

Was erfahren Kinder und Jugendliche im Religionsunterricht über den Globalen Süden? Die Religionspädagogik betont zunehmend die globale Perspektive religiöser Bildung. Als interdisziplinäres Fach verknüpft sie ihre Forschung unter anderem mit der Kulturwissenschaft und den Erziehungswissenschaften und reflektiert auch mit ihrer Hilfe ihre eigenen Bildungsmedien. Dieser Beitrag legt den Fokus auf das Religionsbuch
„Kursbuch Religion“ in seinen Ausgaben von 1976-2017. Er geht der Frage nach, wie der Globale Süden als Makrokosmos in diesem Mikrokosmos repräsentiert wird. Dazu plausibilisiert er zunächst die Sensibilisierung für solche Repräsentationen, um dann die Begriffe „Globaler Süden“, „Postkolonialismus“ und „globalisierte Religion“ im Rahmen der religiösen Bildung zu klären. Das Konzept der Repräsentation wird charakterisiert, bevor das Analysevorgehen, Beispiele sowie diachrone Ergebnisse präsentiert werden. Zuletzt bietet der Artikel Anregungen für die (religiöse) Bildung und das Globale Lernen.

\section{Sensibilisierung für Repräsentationen des Makrokosmos "Globaler Süden"}

Religiöse Bildung in einer globalisierten Welt findet unter anderem mithilfe von Repräsentationen statt. Diese bilden oft als Diskursnetze eine Wissensbasis. Besonders in Lernräumen mit globaler Dimension sind Repräsentationen wirkmächtig. Drei mögliche Situationen in Bezug auf den Globalen Süden im Religionsunterricht verdeutlichen diese These:

- Ein in den Religionsunterricht mitgebrachtes Materialheft soll den südamerikanischen Kontinent und die dortige Armut und Ungerechtigkeit repräsentieren: ein Heft zur globalen Verantwortung.

- Ein im Religionsbuch abgedrucktes Foto von einer Taufe an einem Wasserloch in Nigeria soll im Religionsunterricht die christliche Taufpraxis repräsentieren: ein Foto zum globalen Christentum.

- Ein kurzer YouTube-Clip über Indien soll im Religionsunterricht den fernen Hinduismus und Buddhismus repräsentieren: ein Videoclip zu den globalen Religionen.

Diese drei zugespitzten Beispiele veranschaulichen, wie viel Lerninhalt Repräsentationen zugemutet bzw. aufgelastet werden kann und wie viel Macht sie gleichzeitig in sich tragen. Zugängliche Mikrokosmen sollen den unfassbaren Makrokosmos vereinfachen und erlernbar machen. 
Repräsentation kann demnach als eine pädagogische Kategorie verstanden werden, die eng mit dem Wissensbegriff und dem Verständnis von Bildung verwoben ist. Was erfahren junge Menschen wirklich über Südamerika, wenn sie in einer Schulstunde monoperspektivische, im Globalen Norden verfasste Texte über die Armut der Menschen im Globalen Süden lesen - wohlmöglich ohne historisches Kontextwissen über die Kolonialzeit? Was wissen sie von der globalen christlichen Kirche, die vor allem im Globalen Süden immer mehr Mitglieder gewinnt und längst eigene Theologien und Praktiken entwickelt, wenn sie nur ein Foto einer Taufe in Nigeria betrachten? Was lernen sie über die höchst unterschiedlichen Traditionen des Hinduismus und Buddhismus, wenn sie einen YouTubeClip schauen, der für ein paar Minuten exotische Bilder und Klänge aus Indien ins Klassenzimmer bringt? Alle drei Fälle zeigen, wie Repräsentationen (konstruierte Wirklichkeiten in Form von Artefakten, Texten, Bildern etc.) Wahrnehmungsschemata formen, bestätigen und weiterführen können und religiöse Bildung entscheidend prägen. Postkoloniale Theorien setzen mit ihrer Kritik bereits bei der westlichen Wahrnehmungsweise an, wenn sie Repräsentationen bemängeln. Im postkolonialen Kontext geht es vor allem darum, koloniale bzw. neokoloniale Repräsentationen zu erkennen und zu verlernen, um keine kolonialen Diskurse zu reproduzieren. Wichtig zu verstehen ist, dass Repräsentationen an sich nicht negativ oder zu vermeiden sind, sondern in der Tat notwendig und wichtig sind, um Bildungsprozesse - auch im Bereich des Globalen Lernens - anzuregen.

Der Fokus dieses Beitrags liegt auf dem Makrokosmos Globaler Süden und seinen Repräsentationen im Mikrokosmos Religionsbuch. Warum aber wird ein Religionsbuch, in diesem Fall das „Kursbuch Religion“ (Calwer/Diesterweg-Verlag), zum Gegenstand der Analyse von Repräsentationen des Globalen Südens? Die Untersuchung konzentriert sich auf drei Dimensionen globalisierter Religion. Erstens weitet sie deshalb den Fokus auf den Globalen Süden aus, weil sich die globale Verantwortung des Christentums auf den gesamten Erdball bezieht. Die Folgen der Kolonialisierung und Missionierung sind besonders im Globalen Süden sichtbar und betreffen verstärkt auch den Globalen Norden, z. B. durch Migrationsbewegungen und die fortschreitende religiöse Pluralisierung. Zweitens hat sich der Schwerpunkt des globalen Christentums weltweit betrachtet gen Süden verlagert. Das Christentum erfährt dort einen großen Zuwachs an Gläubigen und neuen Glaubensformen. Drittens sind auch andere globale Religionen, wie sie im Globalen Süden lebendig sind, in ihrer Ausbreitung und Wirkungsweise für die interreligiöse Bildung zu verstehen. Dieser Wandel auf globaler Ebene verlangt nach einer Auseinandersetzung auf der Ebene des Lokalen. Das Lokale ist beispielsweise der Religionsunterricht vor Ort in Deutschland.

\section{Der Globale Süden, Postkolonialismus und globalisierte Religion in der religiösen Bildung}

Der Begriff „Globaler Süden“ erscheint als Pendant zum „Globalen Norden" und ist noch recht jung. Er wird auch von Wissenschaftlerinnen und Wissenschaftlern aus den mit dem Begriff bezeichneten Ländern verwendet und erfährt dadurch eine
Bedeutungsrelativierung (Mignolo, 2011, S. 165-188). Trotzdem ist die Bezeichnung mit der Dominanz des nördlichen Wissens über den Süden konnotiert. Konzeptuelle und theoretische Herangehensweisen werden verwendet, um den Süden einzugrenzen und zu erklären. Der Globale Süden ist - im Unterschied zum geographischen Süden - als eine soziologische Größe zu verstehen, die jene Gebiete meint, die auf dem Index der „menschlichen Entwicklung“ mit einem Bruttoinlandsprodukt pro Kopf bei unter zwei US-Dollar pro Tag liegen. Mit dieser Definition als Grundlage gehören auch Länder des geographischen Nordens zum Globalen Süden und umgekehrt Länder des geographischen Südens zum Globalen Norden. In den entwicklungspolitischen Diskurs wurde die Zuschreibung „Globaler Süden“ Ende der 1980er Jahre vermutlich zuerst von der Weltbank eingeführt. Gleichzeitig dazu etablierte sich der Begriff „Globaler Norden“ (Estermann, 2017, S. 46, S. 205). Bezieht man sich auf Kontinente, so umfasst der Globale Süden überwiegend Afrika, Südostasien und Südamerika. Insbesondere interessieren hier diejenigen Länder, die im globalen Vergleich ökonomisch benachteiligt sind, und zwar aufgrund ihrer Kolonisierung durch ein Land bzw. andauernden Abhängigkeit von diesem Land, das heute dem Globalen Norden zugeordnet wird. Zu betonen ist, dass nicht jedes Land im Globalen Süden eine ehemalige Kolonie ist. Jede der ehemaligen Kolonien hat zudem ihre eigene Geschichte, weshalb die Bezeichnung „Globaler Süden“ in seinem performativ homogenisierenden Effekt durchaus kritisiert werden kann. Im Unterschied zu Begriffen wie „Entwicklungsländer“ oder „Dritte Welt“, die in großem Maße mit Konnotationen aufgeladen sein können, bietet die gedankliche Einheit "Globaler Süden“ jedoch eine weitaus neutralere und denotativ angelegte soziologische Bezeichnung für jene Gebiete der Erde. Im postkolonialen Diskurs geht man von einem dualistischen Raumverständnis aus, welches im Kolonialismus zur Unterordnung und Marginalisierung des kolonialen Anderen führte (Lossau, 2012, S. 355). So spricht Edward Said von „geographischer Gewalt“ (Said, 1994, S. 305), die Ordnung und Kontrolle impliziert, wie sie z. B. konkret in Handlungen wie dem Kartieren und Kartographieren, in Grenzziehungen und Benennungen während der Kolonialzeit durchgesetzt wurde. Dabei war die Praxis der Repräsentation jener „fremden“ Welt ohne Besitzergreifung kaum denkbar (Weimann, 1997, S. 10).

Im religionspädagogischen Diskurs sind Bezüge auf die globale Dimension von Religion und auf den Globalen Süden zu beobachten. In Anlehnung an Simojoki lässt sich religiöse Bildung unter globalisierten Bedingungen aus christlich-theologischer Perspektive in drei Dimensionen erfassen: hinsichtlich globaler Verantwortung, hinsichtlich des globalen Christentums und hinsichtlich globaler Religionen (2012, S. 366 f.). Die christliche Verantwortung umfasst dem christlichen Verständnis nach die ganze Welt, das Christentum ist eine Religion mit globaler Verbreitung (mittlerweile lebt nur noch ein Viertel der Gläubigen im Globalen Norden, drei Viertel im Globalen Süden) sowie auch andere große Religionen wie das Judentum, der Islam, der Hinduismus und der Buddhismus. Bei diesen drei religionsbezogenen Dimensionen bestehen Überlappungen mit Diskursen um den Globalen Süden und Bildung. Besonders die Dimension "globale Verantwortung“ ist eng mit dem globalen Lernen verbunden, das „als pädago- 
gische Reaktion auf die Entwicklungstatsache der Weltgesellschaft, als Reaktion auf die veränderten Lernanforderungen im Zuge der Globalisierung“ (Scheunpflug, 2001, S. 87) verstanden werden kann. Die Dimension „globales Christentum“ ist mit dem ökumenischen Lernen assoziiert und die Dimension „globale Religionen“ mit dem interreligiösen Lernen. Man kann von einer globalen Ausdifferenzierung des Christentums und folglich der christlichen Theologien sprechen, wobei vor allem im Globalen Süden ein starker Zuwachs von Gläubigen $\mathrm{zu}$ verzeichnen ist. Dabei rücken die historischen Verflechtungen und kulturellen Einwirkungen des Christentums auf den Globus ins Blickfeld. Zusammen mit Scheunpflug weist Simojoki auf Ausblendungen hin, die die gegenwärtige Verengung auf das Verständnis von der Diskussion um die Ökumene auf eine „kleine (interkonfessionelle) Ökumene“ (Simojoki \& Scheunpflug, 2016, 146) mit sich bringt. Sie betonen für die ökumenische Bildung im Sinne von Ernst Lange, die Wachstumszentren Afrika, Asien und Lateinamerika - also die Erdteile des Globalen Südens - mit ihren konfessionell spezifischen Ausprägungen des Christentums einzubeziehen.

Nicht nur das Christentum, sondern alle größeren Religionen globalisieren sich. Berücksichtigt man die stets vorhandene kulturelle Einbettung und Verknüpfung von Religion mit Kultur, so spielt die lokale Verortung von Religion auch in globalen Diskursen eine nicht zu unterschätzende Rolle. Dies führt zu großen Herausforderungen für die interreligiöse Bildung, die für diese Dynamik und Komplexität entsprechende didaktische Konzepte entwerfen muss.

\section{(Postkoloniale) Repräsentationen im Bildungsgeschehen}

Noch einmal sei betont: (Religiöse) Bildung in einer globalisierten Welt findet unter anderem mithilfe von Repräsentationen statt. Dabei sind die Merkmale von Repräsentationen zu erfassen und Repräsentationen im Bildungsprozess zu reflektieren, besonders hinsichtlich (post-)kolonialer Muster und Inhalte. Das Repräsentationsgeschehen ist vielschichtig, vielseitig und umfasst mehrere Teilprozesse. Aus der Sekundärliteratur zum Repräsentationsbegriff können folgende Merkmale extrahiert werden: Repräsentation meint Konstruktion (Jamme \& Sandkühler, 2003, S. 16f.), Stellvertretung, Vorstellung, Imagination und Selbstrepräsentation (Weimann, 1997, S. 21). Verbunden ist der Begriff außerdem mit der Idee der Chronologie von ontologischem Original und Repräsentation. Repräsentation beinhaltet Reproduktion, Repetition und somit auch Imitation (Jamme \& Sandkühler, 2003, S. 26f.). Dies kann bis zur Substitution führen, sodass das Original selbst überflüssig wird, denn die Repräsentation hat einen Eigenwert erlangt und wirkt stellvertretend (Thomas, 2011, S. 334). Versteht man jede Repräsentation jedoch als Konstrukt bzw. konstruierte Wirklichkeit, so verlischt die Existenz irgendeines „Originals“. Es gibt dann kein Nacheinander, sondern nur ein Aufteilen bzw. Verteilen von Bedeutung. Darin zeigt sich die Willkür von Bedeutungszuschreibungen. Impliziert sind dadurch Momente der Aneignung und der statischen Abgrenzung, die sich in Identifikationen, Stereotypisierungen und Othering-Prozessen niederschlagen können. Zentraler Auslöser für das Entstehen aller Repräsentationen ist die Intentionalität (Sandkühler,
2009, S. 81-83): Worauf fällt der Blick? Was möchte man sehen, was wie konstruieren und festigen und was wie de-konstruieren und verflüssigen? In diesen Prozessen wirken Machtinteressen. Abhängig vom Ziel bzw. Verwendungszweck einer Repräsentation, finden manche Merkmale eine stärkere Ausprägung oder Betonung als andere. Insgesamt sind alle miteinander verknüpft. So schwingt bei jeder Repräsentation die Selbstrepräsentation, das Selbstbild mit. Beispielsweise haftet kolonialen Repräsentationen in hohem Maße das eurozentrische Selbstverständnis an.

Repräsentation ist jedoch keine „Einbahnstraße“ (Riese, 1997, S. 335). Es gibt Gegenstimmen und Gegenrepräsentationen aus dem Globalen Süden, die koloniale Repräsentationen infrage stellen, indem sie beispielsweise andere Narrative zum gängigen bieten. Es gilt, einem ganzen Diskurs der Hegemonie Widerstand zu leisten, die eigene Stimme zurückzufordern und eine eigene Identität zu entwerfen. Stuart Hall spricht in diesem Zusammenhang von counter-strategies, durch die die Repräsentationen eines dominierenden Regimes in der Tat herausgefordert und verändert werden können. Doch wo lässt sich ein Ansatzpunkt dafür finden? Das Potenzial steckt in der folgenden Annahme: „Meaning can never be finally fixed“ (Hall, 2000, S. 270). Im Rahmen von Bildung sind weitere Aspekte von Repräsentationen zu beachten. So trägt die Aufmerksamkeit zum Rezeptionserfolg bei. Die Dimension der Verantwortung auf Seiten der Repräsentierenden, aber auch auf Seiten der Rezipierenden, spielt eine große Rolle. Sie sind mit am (Miss-)Erfolg der Repräsentationen beteiligt und daran, ob Repräsentationen kolonial oder postkolonial gedeutet und reproduziert werden. Es ist außerdem die Vermittlung von Wissen über Repräsentationsmechanismen nötig, um Lernoptionen nutzen zu können.

\section{Repräsentationen des Makrokosmos Globaler Süden im Mikrokosmos Religionsbuch}

Unterschiedliches Wissen, unterschiedliche Deutungen und Interpretationen werden bei der Produktion eines Schulbuchs machtvoll ausgehandelt und verdichten sich als Konstrukte im Schulbuch. Um für das Globale dieser Repräsentationen zu sensibilisieren, eignen sich postkoloniale Theorien als kulturtheoretisches Analyseinstrument. Die von Thomas Höhne entwickelte Thematische Diskursanalyse (Höhne, 2008, S. 423454) bietet dabei eine geeignete Methode, um die globale Dimension von religiöser Bildung mithilfe von Repräsentationen zu untersuchen. Dabei wird davon ausgegangen, dass niemals der gesamte Diskurs (z. B. um den Globalen Süden) erfasst werden kann, jedoch einzelne Schulbuchseiten als Mikrorepräsentationen des Diskurses Aufschluss über seine typischen Strukturen geben. Fragt man nach der Relationierung von Religion(en) und Religiosität und Globalität in Bezug auf Bildungsprozesse, so kann die Idee vom Lernen anhand von einem lokal zugänglichen Mikrokosmos, in dem der globale Makrokosmos repräsentiert wird, weiterhelfen.

Ein solcher religionspädagogischer Mikrokosmos, in dem sich Diskurse manifestieren, produziert oder auch dekonstruiert werden, ist das Religionsbuch. Wie notwendig eine kritische Untersuchung von Schulbüchern auf koloniale Mus- 
ter hin ist, wurde - auch in internationalen Vergleichen - bereits für andere Fächer nachgewiesen. Für das Fach Evangelische Religion wurde das „Kursbuch Religion“ (Calwer/ Diesterweg-Verlag) in seinen Ausgaben von 1976-2017 für die Jahrgangsstufen 5/6, 7/8 und 9/10 auf die Frage hin untersucht, wie der Globale Süden dort repräsentiert wird. Diese Religionsbücher wurden und werden als Unterrichtswerke für die Haupt-, Realschulen und Gymnasien konzipiert. Dabei ist das „Kursbuch Religion“ „das meistverkaufte und damit erfolgreichste Religionsbuch der letzten 40 Jahre und eignet sich darum im besonderen Maße als Spiegelbild der Religionspädagogik" (Herrmann, 2012, 17). Insgesamt 18 Kursbücher wurden in der vorliegenden Untersuchung analysiert:

\begin{tabular}{|c|c|c|}
\hline Titel der Ausgabe & $\begin{array}{l}\text { Band für die } \\
\text { Jahrgangsstufe }\end{array}$ & $\begin{array}{l}\text { Erscheinungs- } \\
\text { jahr }\end{array}$ \\
\hline \multirow{3}{*}{ Kursbuch Religion } & $5 / 6$ & 1976 \\
\hline & $7 / 8$ & 1978 \\
\hline & $9 / 10$ & 1979 \\
\hline \multirow{3}{*}{$\begin{array}{l}\text { Das neue Kursbuch } \\
\text { Religion }\end{array}$} & $5 / 6$ & 1984 \\
\hline & $7 / 8$ & 1986 \\
\hline & $9 / 10$ & 1988 \\
\hline \multirow{3}{*}{$\begin{array}{l}\text { Kursbuch Religion } \\
\text { Neuausgabe }\end{array}$} & $5 / 6$ & 1995 \\
\hline & $7 / 8$ & 1991 \\
\hline & $9 / 10$ & 1993 \\
\hline \multirow{3}{*}{$\begin{array}{l}\text { Kursbuch Religion } \\
2000\end{array}$} & $5 / 6$ & 1997 \\
\hline & $7 / 8$ & 1998 \\
\hline & $9 / 10$ & 1999 \\
\hline \multirow{3}{*}{$\begin{array}{l}\text { Das Kursbuch Religion } \\
1,2,3\end{array}$} & $5 / 6$ & 2005 \\
\hline & $7 / 8$ & 2005 \\
\hline & $9 / 10$ & 2007 \\
\hline \multirow{3}{*}{$\begin{array}{l}\text { Neuauflage Kursbuch } \\
\text { Religion } 1,2,3\end{array}$} & $5 / 6$ & 2015 \\
\hline & $7 / 8$ & 2016 \\
\hline & $9 / 10$ & 2017 \\
\hline
\end{tabular}

Tab. 1: Übersicht über die untersuchten Schulbücher der vorliegenden Untersuchung, Quelle: eigene Darstellung

Die konkreten Ergebnisse der Thematischen Diskursanalyse werden nun hinsichtlich der drei Dimensionen mithilfe von Analysebeispielen veranschaulicht.

\section{Globale Verantwortung}

Ein Analysebeispiel (Jahrgangsstufe 9/10, 1979, S. 219) zeigt, dass Repräsentationen des Globalen Südens beim Thema Kolonialismus und Mission besonders als Konstruktionen auftauchen, bei der ein Gegensatz von überlegenem Globalem Norden und hilfsbedürftigen homogenem Globalen Süden (dort bezeichnet als „Dritte Welt") aus Kolonialzeiten reproduziert wird. Als Folge der Asymmetrie wird der Topos der notwendigen Solidarität bzw. Entwicklungshilfe eingeführt. Aus postkolonialer Perspektive werden auf diese Weise koloniale Abhängigkeitsverhältnisse fortgeführt. Die diachrone Analyse zeigt, dass Repräsentationen des Globalen Südens gerade in der Dimension "globale Verantwortung“ auch deutliche Selbstrepräsentationen des Globalen Nordens sind, der vor allem als helfende Instanz bezüglich der verschiedenen Notlagen im Globalen Süden dargestellt wird. Das Christentum dient dabei als Motivation, Begründung und Instrument. Mit der vierten Ausgabe (1997-1999) beginnt nach einer Viktimisierung des Globalen Südens eine neue Phase, in der (parallel dazu) die Eigendynamik des zunehmend als heterogen repräsentierten Globalen Südens gezeigt wird. Die Anzahl und Differenzierung der Themenbereiche, in denen Repräsentationen des Globale Süden in der Dimension „globale Verantwortung“ zu finden sind, nehmen merklich zu, vor allem bezogen auf die jeweiligen Bände für die Jahrgangsstufe 9/10.

\section{Globales Christentum}

Ein analysiertes Beispiel (Jahrgangsstufe 5/6, 1984, S. 138) thematisiert die Taufpraxis am Beispiel von Nigeria. Es wird ein Typus als Stellvertretung konstruiert und der Topos der Unterentwicklung bedient. Der Fokus wird allerdings auf die ökumenisch verbindende Taufpraxis gelegt. Aus postkolonialer Sicht kann kritisiert werden, dass im Beispiel dem Entwicklungsparadigma des Westens gefolgt wird und Nigeria einseitig repräsentiert wird. Diachron betrachtet weisen die Repräsentationen des Globalen Südens besonders in der Dimension „globales Christentum" große Unterschiede auf. Verschiedene Schwerpunktsetzungen und Entwicklungsschübe sind durch die diachrone Analyse der Kursbücher deutlich geworden. Die Thematisierung des Christentums bezieht sich anfangs eher lokal auf Deutschland. Repräsentationen in der Dimension "globales Christentum“ werden oft von dem Thema Mission (heute) abgeleitet. Geht es um Befreiungstheologie und den Kampf gegen Ungerechtigkeit in ehemaligen Kolonien, dann überlagert dies oft den global verbindenden ökumenischen Aspekt. Überlappungen der Diskurse zu „globaler Verantwortung" und "globalem Christentum" sind zu beobachten. Im Rahmen zentraler Elemente des christlichen Glaubens wird der Globale Süden repräsentiert - am beliebtesten sind dabei Fotos von Taufen. Die Authentizität von Repräsentationen aus dem Globalen Süden und die tatsächliche Sprecherintention bei Textdokumenten sind teilweise unersichtlich.

\section{Globale Religionen}

Ein Analysebeispiel (Jahrgangsstufe 7/8, 1991, S. 157) zum Thema Mission und Dritte Welt (Tamilen) verdeutlicht, wie die Dimensionen „globale Verantwortung“ und „globale Religionen " zusammenhängen bzw. als hier zusammenhängend repräsentiert werden. Indien wird dabei mit indischen Tamilen repräsentiert, die exemplarisch für „das Heidentum in Indien“ stehen. Eine asymmetrische Repräsentationsweise ist daran erkennbar, dass historische Repräsentationen von indischen Tamilen und zeitgenössische Repräsentationen von Christen parallelisiert werden. Imaginationen über die Tamilen werden geweckt und der Topos der Andersartigkeit durchzieht die Einheit. Auch wenn ein heterogenes Bild der Tamilen präsentiert wird, fehlt im postkolonialen Sinne eine Stimme der Tamilen selbst aus jener Zeit oder auch rückblickend von heute. 
Bei den Repräsentationen des Globalen Südens in der Dimension "globale Religionen" sind vorrangig die großen thematischen und quantitativen Differenzen in den jahrgangsbezogenen Bänden im diachronen Vergleich offensichtlich. Monoton finden sich in den jeweiligen Bänden für die Jahrgangsstufen 5/6 und 7/8 dieselben Themenbereiche oder gar keine, in denen der Globale Süden repräsentiert wird. Der Globale Süden ist bis zum Jahr 2000 in den Bänden für die beiden unteren Jahrgangsstufen meist in Bilddokumenten oder bei einer historischen Verortung von Religionen und religionskundlichen Informationen relevant. Bis zur sechsten Ausgabe (2015-2017) ist der Globale Süden im Unterschied zu den anderen beiden Dimensionen in der Dimension ,globale Religionen " insgesamt stark unterrepräsentiert. Ab der dritten Ausgabe (1991-1995) werden die Themenbereiche und Repräsentationsmuster für die Jahrgangsstufe 9/10 differenzierter und sind nicht mehr nur noch auf die „entfernten" Religionen Hinduismus und Buddhismus begrenzt. Religionsübergreifende Diskurse, in die Repräsentationen des Globalen Südens einbezogen werden, gewinnen an Bedeutung. Abgesehen davon ist zu beobachten, dass Religionen in den Religionsbüchern bis einschließlich zur vierten Ausgabe (1997-1999) unabhängig voneinander und wenig interreligiös vorgestellt werden. Auch wenn nicht nur eurozentrische Perspektiven auf den Globalen Süden geboten werden, sind doch immer wieder Othering-Prozesse festzustellen.

Die Ergebnisse der Thematischen Diskursanalyse bieten einen diachronen Befund für den Mikrokosmos Religionsbuch bezüglich der Relationierung von Religion(en) und Globalität in der religiösen Bildung. Im Hinblick auf die diachronen Entwicklungen der Repräsentationsarten und der Topoi zeigt sich, dass diese keineswegs in allen drei untersuchten Dimensionen gleichförmig sind, sondern einzeln betrachtet und ausgewertet werden müssen. Es kann keine stereotypisierende Repräsentationspraxis festgestellt werden, wenn es um die Thematisierung des Globalen Südens im „Kursbuch Religion“ in den Ausgaben zwischen 1976 bis 2017 geht. Vielmehr überrascht, wie divers die Prozesse von Ausgabe zu Ausgabe verlaufen. So werden in manchen Bänden mehrperspektivische Repräsentationsmuster geboten und in den nachfolgenden sind wieder vereindeutigende, nur eurozentrische Repräsentationen zu finden. Ebenso verhält es sich mit postkolonialen Reflexionen (z. B. in Einheiten zum Kolonialismus und Mission), die nur in manchen Bänden vorhanden sind. Darüber hinaus wird durch die übergreifende Analyse deutlich, dass die drei Dimensionen nicht streng voneinander zu trennen sind, sondern sich gegenseitig diskursiv überlappen. Es zeigt sich außerdem, dass sich die Perspektive hin zum Globalen zwischen 1976 und 2017 bezogen auf alle Ausgaben dieses Religionsbuchs mehr und mehr weitet.

\section{Anregungen für die (religiöse) Bildung und das Globale Lernen}

Die vorgestellten Ergebnisse der diachronen Untersuchung führen zu Überlegungen, die konkrete Inhalte sowie die Methodik globaler Lernthemen betreffen. Das Konzept der Repräsentation ist bisher kein Thema der religionspädagogischen Forschung, wirkt jedoch in die Religionspädagogik hinein. So werden andere Aspekte, die mit dieser Thematik verknüpft sind, bereits bearbeitet. Es entwickelt sich beispielsweise eine Sensibilität für Globalisierungsprozesse, für Machtfragen, für Wahrheitsansprüche, für die Dekonstruktion von vermeintlichen Wirklichkeiten, Diskursen und Wissen, für Geschichtlichkeit, für Übersetzungsfragen, für Identitätsfragen, für Interkulturalität und andere Herausforderungen durch die Transformation der Religionspädagogik selbst. Dazu gehört auch, dass sich diese Disziplin erst neuerdings durch postkoloniale Theorien bereichern lässt.

Für das Globale Lernen ist es förderlich, die Vielschichtigkeit und Mehrdimensionalität von religiöser Bildung zu beachten. Sowohl bei ethischen, als auch bei ökumenischen und interreligiösen Themen ist die Repräsentationspraxis postkolonial zu reflektieren bzw. zu gestalten. So wird eine differenzierte Wahrnehmung von diesen religionsbezogenen Diskursen und auch von (sich auch überlappenden) Diskursen in andere Bereiche des Globalen Lernens möglich. Das Globale Lernen wiederum kann die Religionspädagogik bereichern durch ihren inhaltlich weiten Fokus und ihre gleichzeitig pädagogische Tiefe.

Es kann konstatiert werden, dass in Schulbüchern, anderen Bildungsmedien und Lernprozessen insgesamt ein Repräsentieren stattfindet und dies sogar zu den Funktionen von Lernmedien gehört. Die Frage nach den „richtigen“ Repräsentationen ist nicht (leicht) zu beantworten - eher geht es darum, Repräsentationen als „richtige“ Antworten in Frage zu stellen.

Die vorangegangenen Erläuterungen über Repräsentationen und postkoloniale Theorien als Analyseinstrumente zur Sensibilisierung ebendieser in der religiösen Bildung weisen ein Anregungspotenzial auch für das Globale Lernen und dortige Repräsentationen von Religion auf. Religionen haben selbst globale Dimensionen sie können nicht als „Originale“ oder Ganzheiten erfasst werden, wie die drei Beispielsituationen zu Beginn des Beitrags verdeutlichen. Eine „Kontextualisierung von Religion vor dem Hintergrund einer globalen Lerndimension" im Rahmen des Globalen Lernens ist daher so nicht möglich, denn Religion selbst beinhaltet eine Lerndimension des Globalen.

Der argentinische Literaturwissenschaftler Walter D. Mignolo spricht von der „dekolonialen Option“, dem Verlernen und Entkoppeln aus der kolonialen Matrix: „learning to unlearn in order to relearn" (2012, S. 165-188). Er benennt damit einen Ausweg für Lernende im Globalen Süden. Aber auch im Globalen Norden kann und sollte das Verlernen eine Option sein, um eine postkoloniale Aufarbeitung in und durch die Bildung zu fördern.

Wenn Konzepte Globalen Lernens den Erwerb von Kompetenzen zur Bewältigung weltgesellschaftlicher Komplexität (Treml, 2011, S. 190-203) betonen, dann rücken Repräsentationstechniken ins Blickfeld. Darüber hinaus kann das Globale Lernen des Globalen Nordens in seiner Thematisierung von Religion(en) insofern bereichert werden, dass es $R e-$ präsentationen von Religion(en) aus dem Globalen Süden aufnimmt. Untersucht werden könnten dazu Bildungsmedien anderer Länder, beispielweise Religionsbücher. Diachrone Analysen dieser Materialien würden helfen, die bisherige dortige Repräsentationspraxis bezüglich Religion(en) zu erfassen. Dann wird auch das Globale Lernen selbst global vervollständigt durch Ansätze aus dem Globalen Süden - Religion kann dabei gerade der Faktor sein, der trotz seiner äußerlichen Komplexität eine innere einfache Verbindung herzustellen vermag. 


\section{Literatur}

Estermann, J. (2017). Südwind. Kontextuelle nicht-abendländische Theologien im globalen Süden. Wien: LIT.

Hall, S. (2000). The Spectacle of the "Other". In S. Hall (Hrsg.), Representation. Cultural Representations and Signifying Practices (4. Edition, S. 223-279). London, Thousand Oaks, New Delhi: Sage Publications.

Herrmann, H. J. (2012). Das Kursbuch Religion - ein Bestseller des modernen Religionsunterrichts. Ein Beitrag zur Geschichte der Religionspädagogik seit 1976. Stuttgart: Diesterweg.

Höhne, Th. (2008). Die thematische Diskursanalyse - dargestellt am Beispiel von Schulbüchern. In R. Keller, A. Hirseland, W. Schneider \& W. Viehöver (Hrsg.), Handbuch sozialwissenschaftliche Diskursanalyse. Band 2: Forschungspraxis (3. Auflage, S. 423-454). Wiesbaden: Springer. https://doi.org/10.1007/978-3-531-92585$1 \_15$

Jamme, Ch. \& Sandkühler, H. J. (2003). Repräsentation, Krise der Repräsentation, Paradigmenwechsel. Skizze eines interdisziplinären Forschungsprogramms. In S. Freudenberger \& H. J. Sandkühler (Hrsg.), Repräsentation, Krise der Repräsentation, Paradigmenwechsel. Ein Forschungsprogramm in Philosophie und Wissenschaften (S. 15-46). Frankfurt a. M.: Peter Lang.

Lossau, J. (2012). Postkoloniale Geographie. Grenzziehungen, Verortungen, Verflechtungen. In J. Reuter (Hrsg.), Schlüsselwerke der Postcolonial Studies (S. 355364). Wiesbaden: Springer. https://doi.org/10.1007/978-3-531-93453-2_26

Mignolo, W. D. (2011). The Global South and World Dis/order. Journal of Anthropological Research, 67(2), 165-188. https://doi.org/10.3998/jar.0521004.0067.202

Mignolo, W. D. (2012). Learning To Unlearn. Decolonial reflections from Eurasia and the Americas. Columbus: The Ohio State University Press.

Riese, U. (1997). Repräsentation, postkolonial. Eine euro-amerikanische Assemblage. In R. Weimann (Hrsg.), Ränder der Moderne. Repräsentationen und Alterität im (post)kolonialen Diskurs (S. 301-354). Frankfurt a. M.: Suhrkamp.

Said, E. W. (1994). Kultur und Imperialismus. Einbildungskraft und Politik im Zeitalter der Macht. Frankfurt a. Main: S. Fischer.

Sandkühler, H. J. (2009). Kritik der Repräsentation: Einführung in die Theorie der Überzeugungen, der Wissenskulturen und des Wissens. Frankfurt a.M.: Suhrkamp.
Scheunpflug, A. (2001). Der Beitrag der Entwicklungspädagogik zum AgendaProzeß. In O. Herz, H. Seybold \& G. Strobl (Hrsg.), Bildung für nachhaltige Entwicklung. Globale Perspektiven und neue Kommunikationsmedien (S. 87-100). Opladen: Leske u. Budrich. https://doi.org/10.1007/978-3-322-93257-0_7

Simojoki, H. (2012). Globalisierte Religion. Ausgangspunkte, Maßstäbe und Perspektiven religiöser Bildung in der Weltgesellschaft. Tübingen: Mohr Siebeck.

Simojoki, H. \& Scheunpflug, A. (2016). Ökumenische Bildung im Horizont des globalen Christentums, In S.Altmeyer, R. Englert, H. Kohler-Spiegel, E. Naurath, B. Schröder, F. Schweitzer (Hrsg.), Menschenrechte und Religionsunterricht. Jahrbuch der Religionspädagogik 32 (S. 144-155). Göttingen: Vandenhoeck \& Ruprecht. https://doi.org/10.13109/9783788731908.144

Thomas, K. (2011): René Girard: Ein anderes Verständnis von Gewalt. In S. Moebius \& D. Quadflieg (Hrsg.), Kultur. Theorien der Gegenwart (2. Auflage, S. 326338). Wiesbaden: Springer. https://doi.org/10.1007/978-3-531-92056-6_35

Treml, A. K. (2011). Globalisierung als pädagogische Herausforderung: Möglichkeiten und Grenzen einer weltbürgerlichen Erziehung. In W. Sander \& A. Scheunpflug (Hrsg.), Politische Bildung in der Weltgesellschaft - Herausforderungen, Positionen, Kontroversen (S. 190-203). Bonn: Bundeszentrale für politische Bildung.

Weimann, R. (1997). Einleitung: Repräsentation und Alterität diesseits/jenseits der Moderne. In R. Weimann (Hrsg.), Ränder der Moderne. Repräsentationen und Alterität im (post)kolonialen Diskurs (S. 7-43). Frankfurt a. M.: Suhrkamp.

\section{Julia Henningsen}

Seit 2016 Gymnasiallehrerin für Englisch und Ev. Religion, 2016-2019 Mitarbeiterin im DFG-Projekt „Die Professionalisierung des Religionslehrerberufs in Westdeutschland zwischen 1949 und 1989" (Prof. Dr. Henrik Simojoki \& Prof. Dr. Friedrich Schweitzer), seit Nov. 2019 Referentin im Bibelzentrum Schleswig; wissenschaftliche Interessenschwerpunkte: Schulbuchforschung im Kontext postkolonialer Theorien, Professionalisierung des Religionslehrerberufs

\section{OUR BOOK RECOMMENDATION}

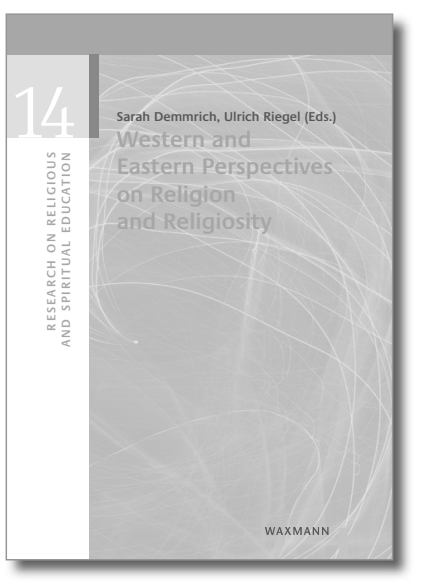

With contributions by Lars Allolio-Näcke, Adnan Aslan, Prashant Bansode, Rito Baring, Dennis S. Erasga, Christel Gärtner, Nihal İşbilen, Hasan Kaplan, Melike Nazlı Kaplan, Ulrich Kropač, Sabrina Müller, Muhamad Supraja, Stephan Winter
Sarah Demmrich, Ulrich Riegel (Eds.)

\section{Western and Eastern Perspectives on Religion and Religiosity}

Research on Religious and Spiritual Education, vol. 14, 2021, 175 pages, br., $€$ 27,90, ISBN 978-3-8309-4306-8, E-Book: € 24,99, ISBN 978-3-8309-9306-3

The concept of religiosity is a highly individual aspect of religion. The understanding ofitwasshaped in Protestantcircles in the Western contextand ithasinspired a huge body of research and further developments in theology, as well as in religious education. However, both charismatic movements within Christianity and orthopractic religious traditions such as Islam raise the question of whether an individualized account of religiosity is able to grasp the spectrum of lived religion comprehensively. Furthermore, with increasing globalization, even Asian worldviews like Hinduism or Buddhism are part of daily experience and have expanded the notion of what can be perceived of as religion. These changes were discussed at the international conference 'Religiosity in East and West: Conceptual and Methodological Challenges' in June 2019. With this volume we pay special attention to the most significant conference contributions relevant to religious education and practical theology. 